\title{
Performance of Postural Balance in Children and Adolescents Living With and Without HIV
}

Rafaela Catherine da Silva Cunha de Medeiros ( $\nabla$ rafaelacath@hotmail.com )

Federal University of Rio Grande do Norte

Racquel Guimarães da Silva

Federal University of Rio Grande do Norte

Radamés Maciel Vitor Medeiros

Federal University of Rio Grande do Norte

Carlos Jean Damasceno de Goes

Federal University of Rio Grande do Norte

Jason Azevedo de Medeiros

Federal University of Rio Grande do Norte

José Angelo Barela

Sao Paulo State University

Breno Guilherme de Araújo Tinoco Cabral

Federal University of Rio Grande do Norte

Paulo Moreira Silva Dantas

Federal University of Rio Grande do Norte

\section{Research Article}

Keywords: Postural balance, HIV, Childhood, Adolescence

Posted Date: February 25th, 2021

DOI: https://doi.org/10.21203/rs.3.rs-237318/v1

License: (c) (1) This work is licensed under a Creative Commons Attribution 4.0 International License. Read Full License

Version of Record: A version of this preprint was published at Gait \& Posture on January 1st, 2022. See the published version at https://doi.org/10.1016/j.gaitpost.2021.09.204. 
1 higher values were observed in the HIV+ group for the predominant ML frequency ( $p=$

$20.04)$ and mean $\operatorname{AP}(p=0.001)$ and ML frequencies $(p=0.001)$. Regarding the evaluation

3 of conditions, a difference was found only in the predominant AP frequency $(p=0.001)$.

4 Higher values were found in the closed eye condition than in with foam $(\Delta=+103 \%)$.

5 The results of the present study indicated that children and adolescents living with HIV

6 have poorer postural control performance than those without HIV.

7

8 Keywords: Postural balance, HIV, Childhood, Adolescence

9 


\section{Introduction}

HIV infections among adolescents are still an ongoing global health problem, despite decreasing by one-third since 2010 . However, progress in adolescents remains slower than that in younger children, which is due to the fact that in different regions, only a few HIV infection prevention programs have been identified as being successful ${ }^{1}$. To confirm transmission trends, information from a group of live births and data from the mothers were analyzed through a time series modeling after the notification of HIV-1 infection cases, and a reduction of $50 \%$ in vertical transmission was observed between 1994 and 2016. Additionally, the prognosis regarding HIV was analyzed for each Brazilian state, the results of which indicated that the Northeast Region of Brazil was one of the areas with the poorest prognosis (increased prevalence of vertical HIV transmission), followed by the Northern, Midwest, Southeast, and Southern regions of Brazil $^{2}$.

HIV infection in children and adolescents represents an even more striking problem, since HIV infection acquired when the immune system is not developed makes children and adolescents more susceptible to the rapid progression of the disease ${ }^{3}$, resulting in distinct chronic clinical complications and causing severe morbidity ${ }^{4}$. There is evidence that HIV acquired perinatally affects neurodevelopment by leading to direct neuronal damage in the fetus ${ }^{5}$, which promotes lower performance in neuropsychological tasks when compared with that of those without HIV ${ }^{6}$.

The deleterious effects of HIV occur due to the neurotropic nature (capable of infecting nerve cells) of the virus. At the onset of infection, the virus can be detected in the cerebrospinal fluid and brain tissue, thereby affecting the central nervous system (CNS) ${ }^{7}$. Such findings are very worrisome, considering that the CNS is the 
1 communication network responsible for sending nerve impulses to the muscles to

2 generate neuromotor responses ${ }^{8}$, controlling mobility and stability ${ }^{9}$.

In contrast, it is known that the treatment of HIV in early childhood can minimize

4

5

6

7

various losses by preserving neurodevelopmental function ${ }^{5}$, as treatment allows for the uninterrupted suppression of viral load and preservation of CD4+ T cells ${ }^{10}$. However, children with HIV, who are treated with highly active antiretroviral therapy (HAART), remain at risk of developing a CNS disease ${ }^{11}$. In addition, the chronic administration of HAART promotes adverse side effects, such as metabolic complications ${ }^{12}$, impaired growth and development ${ }^{13}$, and decreased physical fitness ${ }^{14}$. This knowledge causes great concern, since motor and neurocognitive disorders in pediatric patients can impact various daily functions (e.g., school, leisure, sports) ${ }^{15}$ and increase susceptibility to the deterioration of postural control ${ }^{16}$.

In humans, the postural control system has two behavioral objectives: orientation and balance ${ }^{17}$. Orientation refers to achieving and/or maintaining a given position of the various body segments in relation to the environment. Balance refers to maintaining, reaching, and/or restoring the center of gravity within the support base, therefore balancing the forces acting on the body and its various segments. These two objectives are achieved through a continuous and dynamic relationship between sensory information and muscle contraction ${ }^{17,18,19}$, and through various postural control strategies that can be predictive or reactive ${ }^{20}$.

Despite advances in understanding the functioning of the postural system, little is known about postural control in children with HIV, creating a gap that needs to be filled. As such, the hypothesis of the present study was that children and adolescents with HIV have poorer postural control than their peers without HIV. Therefore, the present study 
1 aimed to evaluate and compare the postural control of children and adolescents with and

2 without HIV, through the performance of various postural tasks.

\section{$4 \quad$ Results}

\section{General features} $\left.\mathrm{t}_{(30)}=2,951 ; p=0.006\right)$.

Table 2 shows the individual information for participants in the HIV+ group. Regarding schooling, a large portion of the participants are in elementary education 1 and 2, and most do not participate in physical education classes; moreover, in the clinical data, it was observed that only two volunteers had CD4 count values below 200 cells $/ \mathrm{mm}^{3}$, which is a warning sign for advanced immunosuppression.

\section{*Insert table 2 here*}

\section{Postural control}

Table 3 shows the results for magnitude, speed, and perimeter of the center of pressure $(\mathrm{CP})$ variation for the groups, as well as the experimental conditions and the respective statistical results. Higher values were observed in the HIV+ group for mean anteroposterior speed $\left(\mathrm{F}_{(1.88)}=16,332 ; p=0.001 ; \eta_{\mathrm{p}}^{2}=0.157\right)$, mediolateral speed $\left(\mathrm{F}_{(1.88)}\right.$ $\left.=43,097 ; p=0.001 ; \eta_{\mathrm{p}}^{2}=0.329\right)$, AP perimeter $\left(\mathrm{F}_{(1.88)}=16,393 ; p=0.001 ; \eta_{\mathrm{p}}^{2}=0.157\right)$, and ML perimeter $\left(\mathrm{F}_{(1.88)}=43,246 ; p=0.001 ; \eta_{\mathrm{p}}{ }^{2}=0.330\right)$. In the evaluation of conditions, a difference significant was observed for AP mean oscillation amplitude $\left(\mathrm{F}_{(1.88)}=3,373 ; p=0.039 ; \eta_{\mathrm{p}}^{2}=0.071\right)$. Post hoc tests indicated that AP MAO was lower 
1 for the condition "with vision" when compared with the conditions "without vision" and

2 "with foam (WF)." No differences were observed for group interaction and condition for 3 any variable.

\section{*Insert table 3 here*}

Table 4 shows the results of the predominant and average frequency of CP variation for the groups and experimental conditions, as well as the respective statistical results. Higher values were observed for the HIV+ group for the predominant ML frequency $\left(\mathrm{F}_{(1.88)}=4,339 ; p=0.04 ; \eta_{\mathrm{p}}^{2}=0.047\right)$ and average $\mathrm{AP}\left(\mathrm{F}_{(1.88)}=17,245 ; p=0.001 ; \eta_{\mathrm{p}}{ }^{2}=\right.$ $0.164)$ and $\mathrm{ML}$ frequencies $\left(\mathrm{F}_{(1.88)}=24,903 ; p=0.001 ; \eta_{\mathrm{p}}{ }^{2}=0.221\right)$. In the evaluation of conditions, a difference significant was found only for the predominant AP frequency $\left(\mathrm{F}_{(1.88)}=9,171 ; p=0.001 ; \eta_{\mathrm{p}}{ }^{2}=0.172\right)$. Post hoc tests indicated higher values in the closed eye condition (CEC) than in with foam $(\Delta=+103 \%)$. No difference was observed for group interaction and condition for any variable.

\section{*Insert table 4 here*}

\section{Discussion}

The present study aimed to compare the postural control of children and adolescents with and without HIV during the performance of various postural tasks. The primary hypothesis was that children and adolescents with HIV have poorer postural control than their peers without HIV. The results of the present study corroborate this hypothesis, and the analyses of the temporal and spectral domains clearly indicated that children and adolescents with HIV have decreased performance compared to children and adolescents without HIV. Surprisingly, no significant differences were observed between the different postural control tasks. Poor performance in these tasks presents important implications for the functioning of postural control in children and adolescents with HIV. Analyses of the temporal domain showed an increase in speed and a larger perimeter for 
1 the ML and AP directions in children and adolescents with HIV, indicating differences in

2 the functioning of postural control in these subjects. Although no differences in MAO

3 were found between the groups, the perimeter values were higher in children and adolescents with HIV. The increased perimeter values in children and adolescents with HIV indicate a greater variation in $\mathrm{CP}$, translating to a difference in capacity to obtain and deterioration of postural control with which to maintain a stable upright position.

The results of the present study corroborate the results of the only prior study involving HIV-infected children ${ }^{16}$, with a greater displacement of the CP. Therefore, the results of the present study add and reinforce this important finding that postural control is deteriorated in children with HIV, and further, add that decreased performance can also be observed in adolescents with HIV. Postural control characterized by greater body sway was also observed in adults with HIV ${ }^{21}$. Thus, considering the scarce results available and the results of the present study, changes in the performance of the postural control system were observed in children ${ }^{16}$, adolescents, and adults with HIV ${ }^{21}$, highlighting the harmful effects of HIV on the functioning of postural control throughout life.

The results of the present study indicated that children and adolescents with HIV perform a greater number of regulatory activities while maintaining an upright position, with $\mathrm{CP}$ variation characterized by higher speed values and higher frequencies (predominant and medium) in the ML and AP directions. These results again corroborate those of the few existing studies regarding children ${ }^{16}$ and adults ${ }^{21}$ with HIV. Moreover, higher values for speed and frequency explain the difference observed for the values of MAO and perimeter of $\mathrm{CP}$ variation. With the case of more frequent adjustments (increased frequency), although the $\mathrm{CP}$ varies with greater speed, the MAO does not necessarily indicate greater variability, as the $\mathrm{CP}$ stays closer to an equilibrium point. Conversely, greater speed and more frequent corrections produce a larger perimeter 
1 because of $\mathrm{CP}$ variation along a longer trajectory, even if it is still contained around the

2 equilibrium point.

It can be inferred from the results of the present study that children and adolescents with HIV experience significant changes in postural control, characterized by variations at higher speeds that imply more frequent corrections to maintain a stable upright position. In doing so, subjects may travel a longer trajectory, but manage to keep $\mathrm{CP}$ closer to equilibrium. These differences in postural control behavior, which indicate changes in the functioning of postural control, may be due to the deleterious effects of treatments or therapies intended to control the HIV infection. Furthermore, adverse effects on the brain occur, which are due to the toxicity potential of these treatments, and inflammation caused by the reactivation and continuous elimination of $\mathrm{HIV}-1$ infection ${ }^{22}$. In this sense, it has been suggested that the postural stability and psychomotor speed of HIV-infected adults are impaired and attributable, at least in part, to compromised infratentorial brain systems (regions with a complex network of arteries that vascularize the cerebellar tissue and brainstem) ${ }^{23}$. Sensory neuropathies are associated with HIV, examples of which include distal sensory polyneuropathy (characterized by the distal degeneration of long axons) due to HIV infection and toxic neuropathy due to antiretroviral therapy (ART), associated with the use of dideoxynucleoside reverse transcriptase inhibitors, particularly zalcitabine, stavudine, and didanosine. These two conditions are phenotypically identical, and together form a common neurological disorder that affects individuals with HIV ${ }^{24}$.

It is important to mention that children and adolescents who have verticallyacquired HIV can experience developmental regression (often indolent), as well as progressive deterioration of neurological development, which is often associated with growth failure and loss of developmental milestones, with manifestations in motor and 
1 mental functions, expressive language, and unsatisfactory educational results (due to HIV

2 encephalopathy) ${ }^{5,25}$. It is also thought that the results of poor postural control in the

3 subjects with HIV are due to impairments suffered during childhood, as it is likely that

4 the CNS was not treated as effectively as the lymphatic system. ${ }^{26}$ Furthermore, HAART

5 helps minimize motor and sensory disorders, which are crucial for postural control ${ }^{17,18}$.

6 Finally, there were no clear differences in the postural control of both groups when

7 performing different tasks to maintain an upright position. It is surprising that adults with

8 HIV depend more on their visual aspects to achieve adequate postural control ${ }^{27}$.

9 Likewise, a greater magnitude of oscillation has been observed with the use of the foam

10 base, causing a reduction in proprioception, which may be compromised in adults with

11 HIV ${ }^{27}$. Thus, the lack of clear and conclusive differences between the various task requirements of the present study needs to be further examined in future studies.

Although there were no differences found between the conditions evaluated, the results of the present study allow us to conclude that children and adolescents with HIV have poorer postural control performance than participants without HIV. The results of the present study are important, because given the changes observed, it is necessary to provide clinical rehabilitation or preventive interventions, such as physical activity programs aimed at children and adolescents with HIV, to alleviate the harmful and unwanted effects of HIV on postural control. The results also suggest that a future longitudinal study would be beneficial, to identify factors, in addition to the clinical ones, that can influence the postural control of children and adolescents with HIV, such as body composition, physical fitness, and physical activity level. 
2 determine the sample number, data from the state of Rio Grande do Norte ${ }^{28}$, with an

3 effect size $=0.80, \alpha=0.05, \beta=0.80$, and a standard error of $5 \%$, were adopted, indicating

4 a sampling power of $0.83^{21}$. The present study was approved by the Research Ethics

5 Committee of the Onofre Lopes University Hospital (CEP/HUOL), under opinion

$6 \quad$ 1.535.531, according to resolution CNS196/96, based on the Declaration of Helsinki 1975

7 and addendum of 2000.

8

\section{9}

\section{Subjects}

The present study included 32 children of both sexes between 6 and 18 years of age: 18 were HIV+ and 14 had a negative HIV serology. All of the HIV+ patients were vertically-infected, received ART before study, and underwent regular clinical followups at the Specialized HIV/AIDS Care Service, which, through contact with a pediatric infectologist, were responsible for explaining the primary objectives and procedures of the study, and obtaining a written consent form from each participants and parentes. All accepted to participate. Additionally, each participant and parentes signed an informed consent form prior to participation in the study. The study design is described in figure 1.

Of the children and adolescents screened for the present study, those with negative responses to the Physical Activity Readiness Questionnaire, active opportunistic lung diseases, congenital heart disease, behavioral changes, and/or disabling cognitive and/or physical limitations, such as gait changes, vestibular changes, amputations, visual impairment, hearing loss, chronic diabetes, and hyperhidrosis, were excluded.

\section{*Insert Figure 1 here*}

\section{Laboratory characteristics}


2 (LABMOV) at the Department of Physical Education of the Federal University of Rio

3 Grande do Norte, Natal. The children and adolescents were accompanied by their

4 guardians. Upon arrival, an introductory period was provided, to acclimate the

5 participants to the laboratory and researchers. The environment remained silent, using

6 voice commands from the evaluators only when necessary for guidance on test

7 procedures. The ambient temperature was maintained at $24-25^{\circ} \mathrm{C}$ with a digital

8 barometer, keeping the subjects comfortable when performing the tests.

\section{Medical history}

Structured medical history evaluations were carried out, including demographic data such as age, sex, education, and physical exercise (yes or no), and clinical data regarding infection, CD4 and CD8 lymphocyte counts, viral load, and time and type of ART (combination medication).

\section{Body mass}

Body composition was assessed through the indirect method of dual-energy radiological absorptiometry. The participants were placed in a supine position on the surface of the device (approximately $190 \times 60 \mathrm{~cm}$ ), passed through the scanner's "arm" in a straight line at a distance of $80 \mathrm{~cm}$, with tracking performed from the head to the feet, and data were analyzed on a microcomputer using the GE Lunar software. For this test, the participants were instructed to fast for 8 hours and wear appropriate clothing. Moreover, all participants were instructed to avoid vigorous physical activity, as well as the consumption of caffeine and/or alcohol, for 24 hours prior to the sessions. 


\section{Postural control}

Participants were asked to maintain an upright position while barefoot, with their lower limbs shoulders' width apart, toes facing forward, and arms loose at the sides of the body, on a force platform (Cefise $\left.{ }^{\circledR}\right)$. The position of the feet was monitored by marking points on the platform with a pencil, and a target $(5 \mathrm{~cm}$ gray circle) was positioned in front of the participant $(1.5 \mathrm{~m})$.

The upright posture was maintained during three experimental conditions: with vision and a fixed base, without vision and a fixed base, and with vision and a foam base. Under the condition with vision and a fixed base, participants maintained their position on the platform base and were asked to fix their gaze on the target. Under the condition without vision, participants maintained their position on the platform and were asked to keep their eyes closed. Under the condition with vision and a foam base, participants maintained the upright position on a foam cushion $(3 \mathrm{~cm}$ high and density $28 \mathrm{~kg} / \mathrm{m} 3$ ) positioned on the base of the force platform, and were asked to fix their gaze on the target. Each attempt lasted 30 seconds, and each condition was repeated 3 times, totaling 9 attempts. The acquisition frequency was $100 \mathrm{~Hz}$, and the order of attempts was randomly assigned by a simple draw carried out for each participant.

The CP was obtained from the force values, in the ML and AP directions and filtered with a digital Butterworth filter, second order, with a cutting frequency of $5 \mathrm{~Hz}$. Subsequently, the temporal domain, MAO, MS, and perimeter variables for each $\mathrm{CP}$ variation direction were obtained. MAO corresponded to $\mathrm{CP}$ variance, and was calculated by subtracting a first-order polynomial from the $\mathrm{CP}$ values and subsequently obtaining the standard deviation. MS indicates how fast the $\mathrm{CP}$ variation occurred, and was calculated by adding the absolute values of the $\mathrm{CP}$ value differences, divided by the total time of each attempt. The perimeter represents the length of the CP trajectory, and was 
1 calculated by obtaining the sum of the $\mathrm{CP}$ values over each attempt. In addition, the

2 predominant frequency and mean $\mathrm{CP}$ variation were also obtained for the ML and $\mathrm{AP}$

3 directions. For this, a fast Fourier transform of CP data was performed (power spectral

4 density estimated by the Welch method, $0.024-\mathrm{Hz}$ resolution, 1,024-point segment, and

$550 \%$ overlap) ${ }^{29}$. The average frequency corresponded to the half-area frequency of the

6 power spectrum for each trial ${ }^{30}$, and the predominant frequency corresponded to the

7 predominant frequency of the spectrum.

8

All procedures were performed using specific routines in MATLAB (MathWorks). The mean for each condition was used for further analysis.

\section{Statistical analysis}

The variables of sample characterization (height, BMI, chronological age) and postural control were considered as dependent variables, separated according to the groups (with and without HIV).

Initially, the normality of the data was analyzed using the Shapiro-Wilk test and the kurtosis and asymmetry values, while the homogeneity of the variances was analyzed using the Levene test, according to Mishra's ${ }^{31}$ recommendations (2019). From this, the results were presented as mean \pm standard deviation (parametric data) and medianinterquartile range (nonparametric data). All postural control variables underwent the logarithmic transformation process (log 10$)$, and after a new analysis, were adjusted to the assumption of normality.

Inferentially, the sample characterization variables underwent the t-test for independent samples to identify any significant differences between the HIV+ and HIVgroups. In relation to the other variables, the use of the MANCOVA test allowed the identification of the level of significance for each group (with and without HIV), 
1 condition (with vision, without vision, with foam), and interaction, considering the

2 chronological age and BMI as covariants of the model. In addition to the significance

3 value $(p)$, the effect size ( $\eta$ p2) and the mean difference between conditions $(\Delta)$ were also

4 identified. When significant values were found for any factor, Bonferroni's post hoc test

5 was used to compare pairs (between and within groups). All steps and assumptions using

6 MANCOVA followed the recommendations of Pituch et al. ${ }^{32}$ (2019). $P$-values $<0.05$

7 were considered significant for all factors.

8

9

\section{References}

1. Unicef. Women: at the heart oh the HIV response for children. (2018).

2. Campos Coelho, V. A., Campos Coelho, F. H., Arraes, L. \& Crovella, S. HIV-1 mother-to-child transmission in Brazil (1994-2016): a time series modeling. Brazilian J. Infect. Dis. 3, 218-223 (2019).

3. Child, T. L. \& Health, A. Editorial Children and youth are crucial in the global AIDS response. Lancet child Adolesc. Heal. 2, 621 (2018).

4. Lowenthal, E. D. et al. Saharan Africa : a review of emerging challenges. Lancet Infect Dis 14, 627-639 (2014).

5. Nielsen-saines, K. Neuroscience and Biobehavioral Reviews Perinatal HIV as an infectious cause of developmental regression. Neurosci. Biobehav. Rev. 102, 417-423 (2019).

6. Haase, V. G., Nicolau, N. C., Viana, V. N., Barreto, G. D. V. \& Pinto, J. A. Executive function and processing speed in Brazilian HIV-infected children and adolescents. Dement Neuropsychol 8, 32-39 (2014).

7. Christo, P. P. Revisão alterações cognitivas na infecção pelo hiv e aids. Rev Assoc Med Bras 2, 242-7 (2010).

8. Duarte, M. \& Freitas, S. M. S. F. Revisão sobre posturografia baseada em plataforma de força para avaliação do equilíbrio. Rev. Bras. Fisioter. 14, 183-192 (2010).

9. Ivanenko, Y. \& Gurfinkel, V. S. Human Postural Control. Front. Neurosci. 12, $1-9$ (2018).

10. Unaids. I Ndetect Ável = I Ntransmi Ssível. (2018). 
1 11. Martin, S. C., Wolters, P. L. \& Toledo-tamula, M. A. Cognitive Functioning in School-Aged Children With Vertically Acquired HIV Infection Being Treated With Highly Active Antiretroviral Therapy ( HAART ). Dev. Neuropsychol. 30, 633-657 (2006).

12. Dimock, D. et al. Longitudinal assessment of metabolic abnormalities in adolescents and young adults with HIV-infection acquired perinatally or in early childhood. Metabolism 60, 874-880 (2011).

13. Almeida, F. J., Kochi, C., Aurélio, M. \& Sáfadi, P. Influence of the antiretroviral therapy on the growth pattern of children and adolescents living with HIV / AIDS. J. Pediatr. (Rio. J). 95, 95-101 (2019).

14. Lima, Luiz Rodrigo Augustemak; Silva, Diego Augusto Santos; Silva, Kelly Samara; Pelegrini, Andreia; Back, Isabela de Carlos; Petroski, E. L. Aerobic Fitness and Moderate to Vigorous Physical Activity in Children and Adolescents Living With HIV. Pediatr. Exerc. Sci. (2017).

15. Tahan, T. T., Bruck, I., Burger, M. \& Cruz, C. R. Neurological profile and neurodevelopment of 88 children infected with HIV and 84 seroreverter children followed from 1995 to 2002. Brazilian J. Infect. Dis. 10, 322-326 (2006).

16. Pontes, L. da S. et al. Variations in plantar pressure and balance in HIV-infected children in antiretroviral therapy. Sci. Rep. 9, 1-7 (2019).

17. Horak, F. B. Adaptation of automatic postural responses. Acquis. Mot. Behav. Vertebr. 57-85 (1996).

18. Barela, J. A., Polastri, P. F. \& Godoi, D. Controle postural em crianças: oscilação corporal e frequiência de oscilação. Rev. Paul. Educ. Física 17, 29-34 (2000).

19. Barela, J. A., Jeka, J. J. \& Clark, J. E. Postural control in children. Exp. Brain Res. 150, 434-442 (2003).

20. Pollock, A. S., Durward, B. R. \& Rowe, P. J. What is balance? Clin. Rehabil. 14, 402-406 (2000).

21. Sounis, E. Bio estatística; princípios fundamentais, metodologia estatística. Aplicação às Ciências Biológicas. in Bio estatística; princípios fundamentais, metodologia estatística. Aplicação às Ciências Biológicas 228 (1971).

22. Veenhuis, R. T., Clements, J. E. \& Gama, L. HIV Eradication Strategies : Implications for the Central Nervous System. Curr. HIV/AIDS Rep. (2019).

23. Sullivan, E. V \& Kemper, C. A. NIH Public Access. Brain Imaging Behav 5, 12 $24(2011)$. 
24. Keswani, S. C., Pardo, C. A., Cherry, C. L., Hoke, A. \& McArthur, J. C. HIVassociated sensory neuropathies. Aids 16, 2105-2117 (2002).

25. Abubakar, A. Infections of the Central Nervous System and Child Development in Sub-Saharan Africa. Handb. Appl. Dev. Sci. Sub-Saharan Africa (2017) doi:10.1007/978-1-4939-7328-6.

26. Almeida, S. M. de. Cerebrospinal fluid analysis in the HIV infection and compartmentalization of HIV in the central nervous system. Arq Neuropsiquiatr 73 (7), 624-629 (2015).

27. Jamison, L., Gines, G., Marshall, J. \& Rosario, M. Vestibular And Proprioceptive Alteration Influence Postural Instability During Dual Tasks In Adults Diagnosed With HIV. J. Student Res. 17, 3283455 (2019).

28. Brasil. Ministério da Saúde. 1-8 (2020).

29. Bonfim, T. R., Grossi, D. B., Paccola, C. A. J. \& Barela, J. A. Additional sensory information reduces body sway of individuals with anterior cruciate ligament injury. Neurosci. Lett. 441, 257-260 (2008).

30. Barela, J. A., Tesima, N., da Silva Amaral, V., Figueiredo, G. A. \& Barela, A. M. F. Visually guided eye movements reduce postural sway in dyslexic children. Neurosci. Lett. 134890 (2020).

31. Mishra, Prabhaker; Pandey, Chandra M; Singh, Uttam; Gupta, A. ; \& Sahu, Chinmoy; Keshri, A. Descriptive Statistics and Normality Tests for Statistical Data. Ann. Card. Anaesth. 20, 456-458 (2019).

32. Pituch, K. A. et al. The Performance of Multivariate Methods for Two-Group Comparisons with Small Samples and Incomplete Data. Multivariate Behav. Res. 0, 1-18 (2019).

\section{Data availability}

The data sets generated during and / or analyzed during the current study are not publicly available due to confidentiality guaranteed to volunteers with HIV, but are available from the corresponding author upon reasonable request.

\section{Acknowledgments}

The authors would like to thank the Giselda Trigueiro Hospital and Natal Specialized Attention Service team for the technical support provided. The Coordenação 
1 de Aperfeiçoamento de Pessoal de Nível Superior - Brasil (CAPES) - gave support for 2 the PhD scholarship (Finance Code 001).

3

4 Conflict of interest

5 The authors declare no conflicts of interest. 


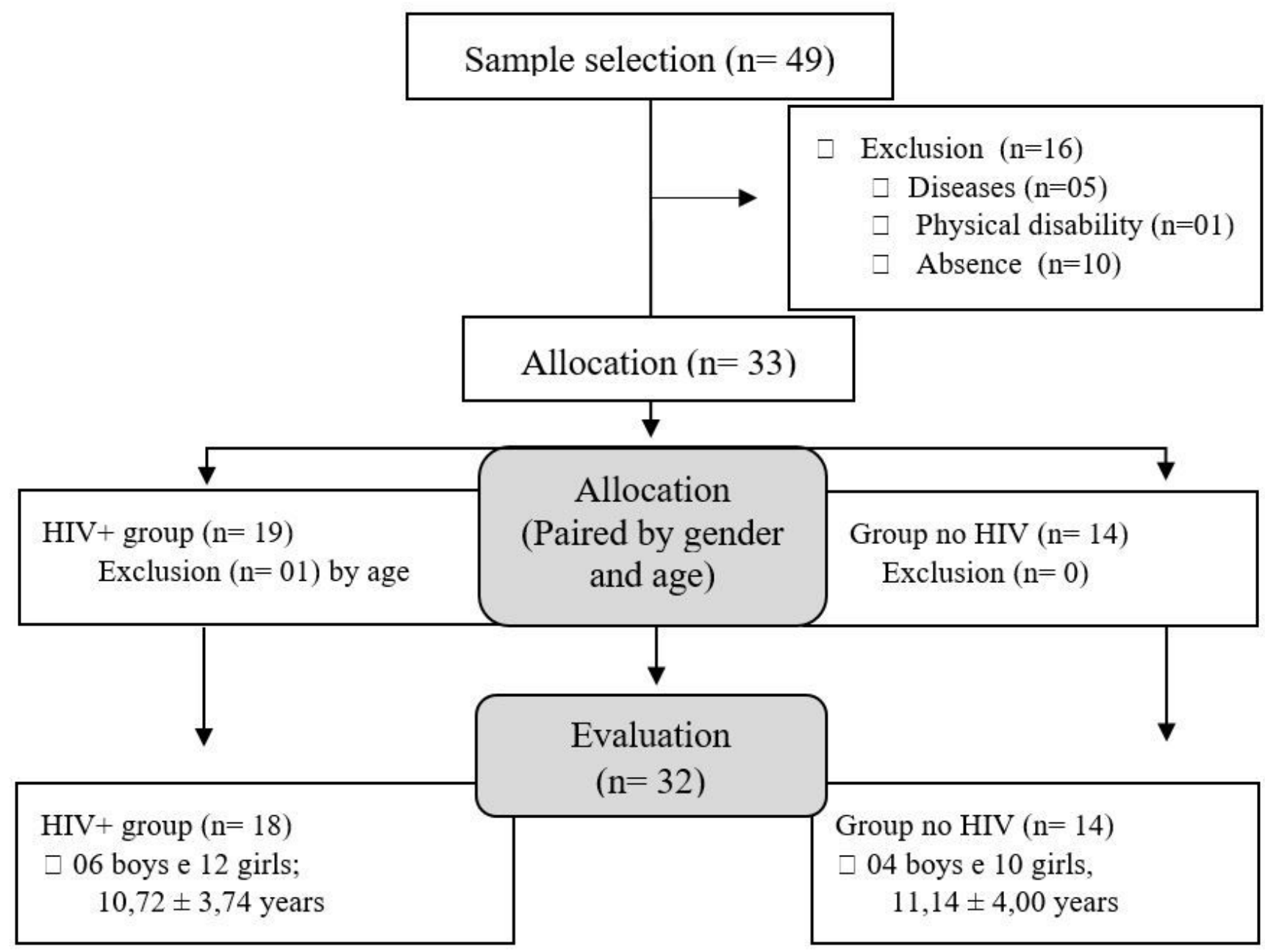

Figure 1

Study flowchart. n: number. 